\title{
Suggested Insulin Regimen for Pregnant Women with Diabetes Who Wish To Fast in Ramadan
}

\author{
Ahmad Shuib Yahaya ${ }^{1,2}$, Adibah Ibrahim ${ }^{2}$, Mohd Shukri Othman ${ }^{2}$, Mohd Pazudin Ismail ${ }^{2}$. \\ ${ }^{1}$ Department of Obstetrics and Gynaecology,Faculty of Medicine \& Health Sciences, Universiti Putra \\ Malaysia. \\ ${ }^{2}$ Department of Obstetrics and Gynaecology,School of Medical Sciences, Health Campus, Universiti Sains \\ Malaysia.
}

\begin{abstract}
Introduction: Pregnant women are among those who are exempted from Ramadan fasting. Despite that, many pregnant women had chosen to fast despite understanding the risk of complications especially hypoglycaemia. In Hospital USM (HUSM), an insulin regime for pregnant women who wish to fast was designed based on expert opinion of obstetricians, but its safety and efficacy are yet to be determined. Objective: To determine the safety and efficacy of the formulated insulin regime using subcutaneous Actrapid $₫$ and Insulatard $₫$ amongst pregnant women with diabetes who fast in Ramadan. Methodology: Pregnant patients with diabetes on insulin who wish to fast during Ramadan were invited to participate in the study. The total daily dose of insulin requirement prior to Ramadan was divided 3 parts; $2 / 3$ for iftar (sunset meal) and $1 / 3$ for sahur (pre-dawn meal). For each timing, $2 / 3$ of the calculated dose was given as short-acting insulin Actrapid $₫$ and remaining $1 / 3$ as intermediate-acting insulin Insulatard $\otimes$. Three patients were monitored in the ward while fasting for two days. Blood glucose checked eight times a day. Following that, eight patients were followed up during Ramadan fasting with this regime. Weekly blood sugar profile (BSP) was taken and glycaemic control evaluated. Results: All patients were able to fast without any hypoglycaemic episode, both during in-patient study and during out-patient Ramadan fasting. Mean daily blood glucose per day for in-patient monitoring was $7.3 \mathrm{mmol} / \mathrm{l}$ with the lowest being $4.56 \mathrm{mmol} / \mathrm{l}$ in the afternoon. During Ramadan fasting, average glucose level was higher $(6.79 \mathrm{mmol} / \mathrm{l})$ compared to prior to Ramadan value $(5.67 \mathrm{mmol} / \mathrm{l})(p>0.05)$. However, improvement of glycaemic control was observed towards end of Ramadan. Conclusion: Pregnant women with diabetes treated with insulin can fast safely during Ramadan using the suggested insulin regime with improvement of glycaemic control observed at the end of Ramadan.
\end{abstract}

KEYWORDS: insulin, gestational diabetes, fasting, Ramadan.

\section{INTRODUCTION}

Up to $5.3 \%$ of deliveries amongst Malaysian population were complicated with diabetes. Out of that, $76.2 \%$ were patients with gestational diabetes mellitus (GDM) on diet control, $19.8 \%$ had GDM on insulin and $4.0 \%$ had pre-existing diabetes. ${ }^{1}$

One of the five pillars of Islam is to fulfil the annual fasting during the holy month of Ramadan. Fasting during Ramadan involves abstaining from food and drink from predawn to sunset for about thirty days. Most people take two meals a day, during Ramadan; sahur (predawn meal) and iftar (sunset meal). The daily fasting period varies from 11 to 20 hours, depending on the geographic location and season. In

Corresponding author:

Ahmad Shuib Yahaya

Department of Obstetrics and Gynaecology,

Faculty of Medicine \& Health Sciences,

Universiti Putra Malaysia.

43400 Serdang

Selangor Darul Ehsan

MALAYSIA

Email: ahmadshuib@upm.edu.my
Malaysia, due to its location near the Equator, Muslims fast for about 13.5 hours each day. ${ }^{2}$

Fasting is not meant to create excessive hardship on the Muslim persons, as clearly mentioned in the Quran and Islamic teachings. Healthy Muslims are obligated to fast during Ramadan, whilst those suffering from illness including diabetes mellitus are exempted from fasting. ${ }^{\text {a }}$

Although they are exempted from fasting, many of those with diabetes still insist on fasting. In the population-based Epidemiology of Diabetes and Ramadan study (EPIDIAR), $42.8 \%$ of patients with type 1 diabetes and $78.7 \%$ with type 2 diabetes from 13 Muslim countries fasted for at least 15 days. ${ }^{3}$ With approximately 1.5 billion Muslims worldwide and the prevalence of diabetes being $4.6 \%$, approximately 55 million people with diabetes worldwide fast during Ramadan.

Studies has shown that majority of healthy pregnant women fast during Ramadan. A cross sectional study of 605 pregnant ladies in Muar District showed $78.84 \%$ of them fasted during pregnancy. ${ }^{4}$ In a study 
in $2004,87 \%$ of Muslim women in Singapore fasted at least one day in Ramadan. ${ }^{5}$ In Pakistan, almost similar observation was made; $87.5 \%$ of ladies fasted during their pregnancies, and almost half of them fasted the whole month of Ramadan. ${ }^{6}$ There had been no survey looking specifically into pregnant women with diabetes with regard to their wishes and practice of fasting during Ramadan. However, experience from our clinical practice shown that majority of them continue carrying out this religious duty without consulting their physicians as they regard themselves as healthy.

The growing concern about the risks of fasting during Ramadan in adults with medical problems, especially diabetes have prompted authorities all around the world, particularly in countries with Muslim majority to formulate and update recommendations and guidelines on management of diabetes during Ramadan. The regularly updated recommendations are made to reduce possible complications, particularly hypoglycaemia during the fasting period.

In general, guidelines formulated for Ramadan fasting had advised pregnant patients with diabetes against fasting, as they are categorized as high risk patients. ${ }^{7,8}$ Recently, a new guideline published by The Malaysian Endocrine \& Metabolic Society and adopted by Ministry of Health Malaysia categorized pregnant women with diabetes in "very high risk" group for Ramadan fasting and they should be advised to abstain from fasting. ${ }^{9}$

In HUSM, back in year 2011, an insulin regimen was designed for pregnant women with diabetes who wish to fast base on the consensus by the obstetricians and gynaecologists. The insulin used are Actrapid®; a short acting insulin, and Insulatard $\AA$; an intermediate-acting insulin. Base on this regime, 2/3 of the total daily dosage of insulin required for Ramadan is administered at iftar and the remaining $1 / 3$ at sahur. For each dose, 2/3 of the required insulin is given as short-acting insulin (Actrapid $\otimes$ ), and another $1 / 3$ is given as intermediate-acting insulin (Insulatard $\AA$ ), as shown in Figure 1.

This regime has been adopted by many other hospitals throughout Malaysia. However, there is no data available with regards to its safety and efficacy. This study was conducted to address these issues.

INSULIN REGIMEN FOR PREGNANT WOMEN WITH DIABETES WHO WISH TO FAST IN RAMADAN

Total daily requirement of insulin during pre-Ramadan; divided into:

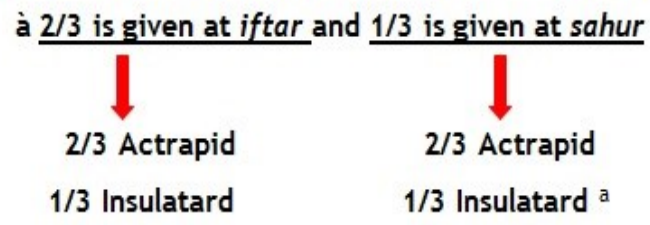

Example of calculation:

Insulin prior to fasting: Actrapid 12 units TDS + Insulatard 18 units ON.

(Total daily insulin dose: 54 units)

Insulin adjustment for fasting during Ramadan:

- For iftar dose: $2 / 3$ of 54 units $=36$ units. $2 / 3$ of 36 units in the form of Actrapid and $1 / 3$ insulatard ( 24 units

Actrapid +12 units Insulatard)

- For sahur dose: $1 / 3$ of 54 units=18 units. $2 / 3$ of 18 units in the form of Actrapid and $1 / 3$ insuatard (12 units actrapid

+6 units insulatard)

So, for Iftar: 24 units of actrapid + 12 units of insulatard and for sahur: 12 units actrapid + 6 units insulatard.

Figure 1: The Hospital USM insulin regime for pregnant women with diabetes during Ramadan fasting

${ }^{a}$ Actrapid $\otimes$ and Insulatard $\otimes$ are listed in the protocol, as those are the type of short-acting and intermediate-acting insulin available in the hospital's pharmacy. 


\section{OBJECTIVE}

The study is designed to evaluate the safety and efficacy of the regime for glycaemic control among pregnant women with diabetes requiring insulin, using the HUSM regime as stated. Since there was no prior control study or proper observation done on the use of this regime, our study could be considered the first observational study to determine the safety and efficacy of this regime.

\section{METHODOLOGY}

Pregnant patients with diabetes who were taking insulin for glycaemic control under antenatal clinic HUSM follow up were invited to participate in this study. The study was done during Ramadan month in 2015. Inclusion criteria include singleton pregnancy, during $2^{\text {nd }}$ or $3^{\text {rd }}$ trimester, and already on insulin injections at least 4 weeks prior to Ramadan month. Exclusion criteria are those on high dose insulin of more than 100 iu per day, patients with diabetic complications of nephropathy, retinopathy, hypoglycaemia unawareness and those with coexisting medical disease such as hypertension, renal disease and epilepsy. Patients with recent diabetic ketoacidosis and severe hypoglycaemia were also excluded, so as patients with mid-trimester detail scan suggestive of foetal anomaly.

The recruitment was carried out four weeks prior to the start of Ramadan month. Antenatal history was obtained and baseline investigations were performed, followed by detail discussion and counselling regarding the study process.

Patients who were recruited into the study were admitted to the antenatal ward. Subcutaneous Actrapid $\circledast$ and Insulatard $₫$ were administrated 30 minutes prior to iftar and sahur according to the regimen protocol in Figure 1. Iftar meals were taken between 19:30- 20:00, depending upon the time of breaking fast, whilst sahur was taken at a fixed time of 05:00- 05:30.

The pre-meal plasma glucose level was taken 30 minutes prior to each meal, just before insulin injection. Subsequent plasma glucose levels were taken at 21:00, 22:00 and 03:00 which are 1 hour, 2 hours and night time post-iftar glucose control. The plasma glucose levels were taken again at 06:00, 07:30, 10:30, 14:30 and 17:30 which are; 1, 2, 5, 9 and 12 hours post-sahur, or day time glucose control to look for any episodes of hypoglycaemia. The same procedure was repeated every day for 2 days before they were discharged. Patients were allowed home on Day 2 if they had no complications. Should any of the patients developed symptoms of hypoglycaemia, they were asked to report to the medical personnel who would determine the capillary glucose level. Patients with glucose level less than $3.9 \mathrm{mmol} / \mathrm{l}$ or those with hypoglycaemic symptom without other recognizable cause were advised to have a meal or sugarcontained fluid.
The patients were seen in the Antenatal Clinic one week after discharge, and weekly thereafter till one week after the end of Ramadan. One day prior to each visit at the Antenatal Clinic, four points capillary sugar profile was taken at 05:00, 08:00, 17:00 and 22:00 which were pre-sahur, post-sahur, pre-iftar and post-iftar respectively.

Patients were asked to record the days of fasting in a calendar and the date and time of hypoglycaemic episodes. They were advised to break their fast by ingesting sugar-contained drinks or food should they experienced the hypoglycaemic episodes. Adjustment of the dosage of insulin at 2 to 4 units was done during the follow-up as per the judgement of the attending doctor or investigator. The target values of glycaemic control were based on recommendation outlined in Malaysian Clinical Practice Guideline, which are $\leq 5.3 \mathrm{mmol} / \mathrm{l}$ for fasting blood glucose and $\leq 6.7 \mathrm{mmol} / \mathrm{l}$ for 2 hours post-prandial blood glucose. ${ }^{10}$

This study had been approved by the Human Research Ethics Committee USM of JEPeM (JEPeM Code: USM/JEPeM/1404133). Informed consent was obtained from all patients before embarking into this study.

All data was analysed using the Statistical Package for Social Science (SPSS) version 22.0. Since the sample was small and skewed, non-parametric tests were used; Mann-Whitney test for independent samples and Wilcoxon Signed Ranks Test for dependent samples. Missing data was handled by Last Observation Carry Forward (LOCF) technique.

\section{RESULTS}

A total of eight patients were recruited into the study. All patients were multiparous. Two of them have GDM, one with type $1 \mathrm{DM}$ and five have type 2 $D M$. The characteristics of the patients were as shown in Table 1.

All patients were on basal-bolus regime of insulin prior to Ramadan fasting. They were on three bolus injections of rapid-acting insulin Actrapid $\otimes$ prior to three main meals; breakfast, lunch and dinner in addition to basal injection of intermediate-acting insulin Insulatard $₫$ during pre-bed around 22:00.

\section{In-Patient Monitoring}

Three out of the eight patients recruited had consented to be monitored in the ward prior to Ramadan fasting. They were admitted separately between one to two weeks prior to Ramadan. During the in-patient study period, all the three patients managed to fast throughout the day in the ward without having any hypoglycaemic symptoms. Figure 2 shows the trend of plasma glucose control of the patient throughout the day. The average plasma glucose level was $7.3 \mathrm{mmol} / \mathrm{l}$. 
Table 1: Demographic data of the patients

\begin{tabular}{|c|c|}
\hline Characteristic & Median (range) \\
\hline Maternal Age (years) & $35.5(15)$ \\
\hline Maternal Weight on booking $(\mathrm{kg})$ & $68.15(35.9)$ \\
\hline $\operatorname{BMI}\left(\mathrm{kg} / \mathrm{m}^{2}\right)$ & $27.2(19.8)$ \\
\hline Gestational Age in beginning of Ramadan (weeks) & $30(13)$ \\
\hline $\mathrm{HbA} 1 \mathrm{C}$ level in early gestation (\%) & $7.3(2.63)$ \\
\hline Average daily Glucose level prior to Ramadan $(\mathrm{mmol} / \mathrm{l})^{a}$ & $5.67(2.30)$ \\
\hline Total Insulin Dose/day prior to Ramadan (i.u.) & $24(42)$ \\
\hline
\end{tabular}

${ }^{a}$ The average was calculated from 4-points BSP readings: (fasting, pre-lunch, pre-dinner and pre-bed)

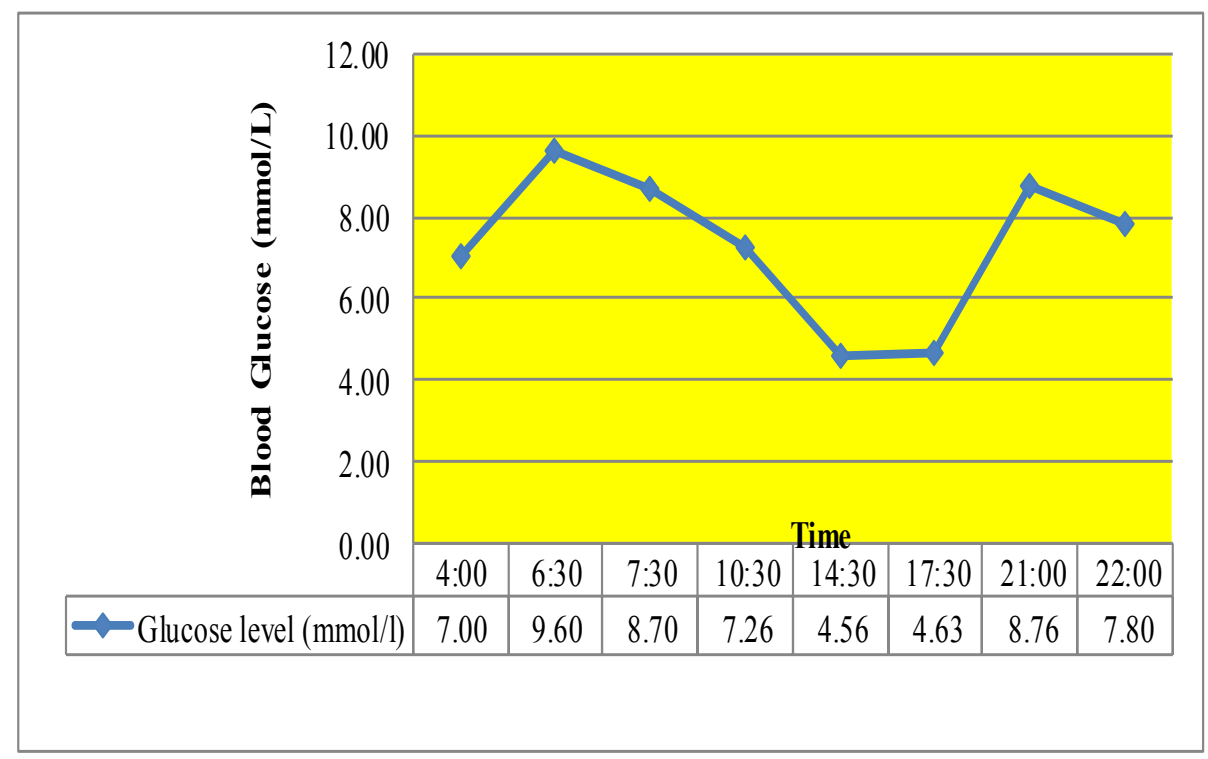

Figure 2: Average Glucose level throughout the day during fasting (in-patient monitoring)

\section{Out-patient Ramadan Fasting Monitoring}

The three patients initially admitted for in-patient monitoring during fasting, together with another five patients were monitored during fasting month of Ramadan. The median days of fasting was 27 days (IqR:9) and no hypoglycaemic symptom reported by all the participants. Seven were able to fast more than 15 days and one fasted the whole month (29 days). The median (IqR) glucose level during Ramadan fasting was higher compared to prior to Ramadan; $6.79 \mathrm{mmol} / \mathrm{l}(0.93)$ and $5.67 \mathrm{mmol} / \mathrm{l}$ (1.13) respectively $(p>0.05)$. The mean plasma glucose level at pre-sahur was noted to be higher than the pre-iftar. Interestingly, the post-sahur and post-iftar mean plasma glucose level were similar, as shown in Figure 3. The weekly plasma glucose level was also been analysed, as shown in Figure 4. It is noted that there was reduction in the level towards the end of Ramadan. Throughout the outpatient monitoring during the fasting period, no blood glucose level less than $3.9 \mathrm{mmol} / \mathrm{l}$ was recorded at any time.

\section{DISCUSSION}

Studies involving the management of pregnant diabetic patients using insulin who insisted on fasting during Ramadan have shown that fasting in this group of patients is generally safe and feasible. ${ }^{11,12}$ However, the insulin regimes used in those studies were not explained. This study found that using the HUSM insulin regime is safe for diabetic pregnant patients who fast.

The average glucose level during Ramadan fasting period was significantly higher as compared to the glucose control prior to Ramadan $(6.58 \mathrm{mmol} / \mathrm{l}$ vs $5.59 \mathrm{mmol} / \mathrm{l}, \quad p<0.05)$. This finding contradicts previous studies done on pregnant women with diabetes who fasted during Ramadan. Azlin et al. (2011) and Ismail et al. (2011) reported reduction in serum fructosamine and $\mathrm{HbA}_{1 c}$ at the end of Ramadan fasting compared to non-fasting level before Ramadan. The differences were that they did not use blood glucose level to assess glycaemic control and the reduction in serum fructosamine 


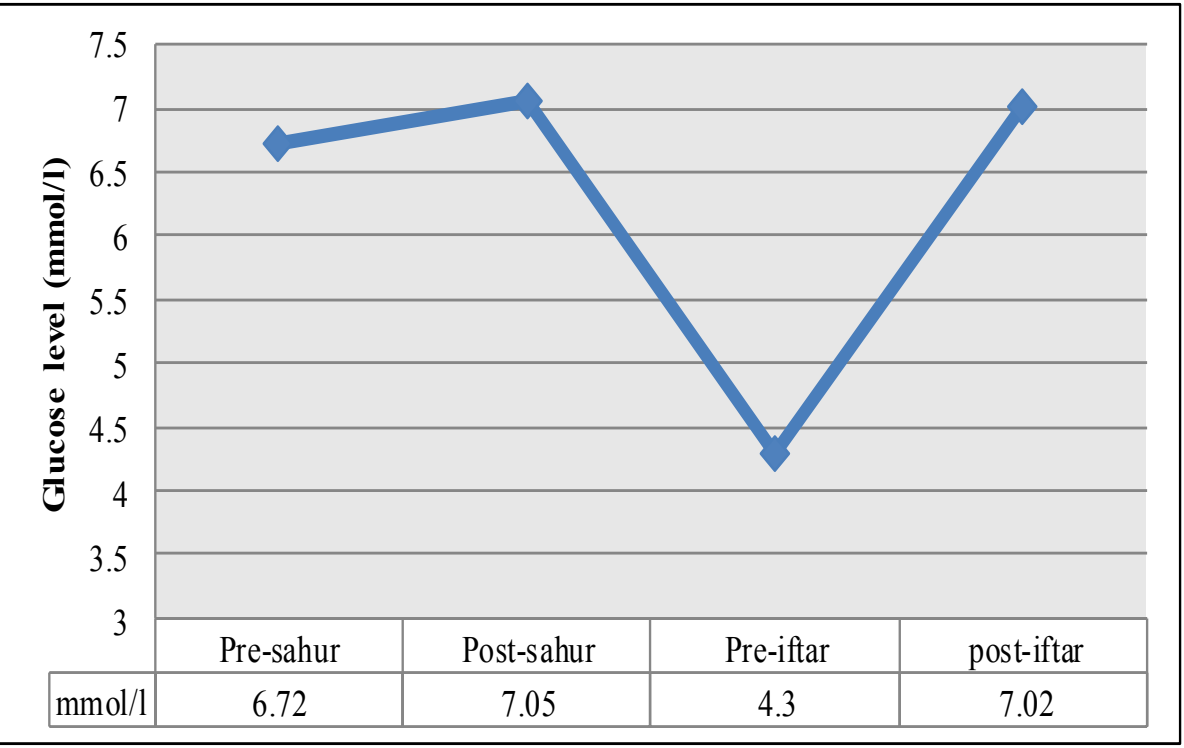

Figure 3: Average Glucose level for each BSP timing (out-patient monitoring)

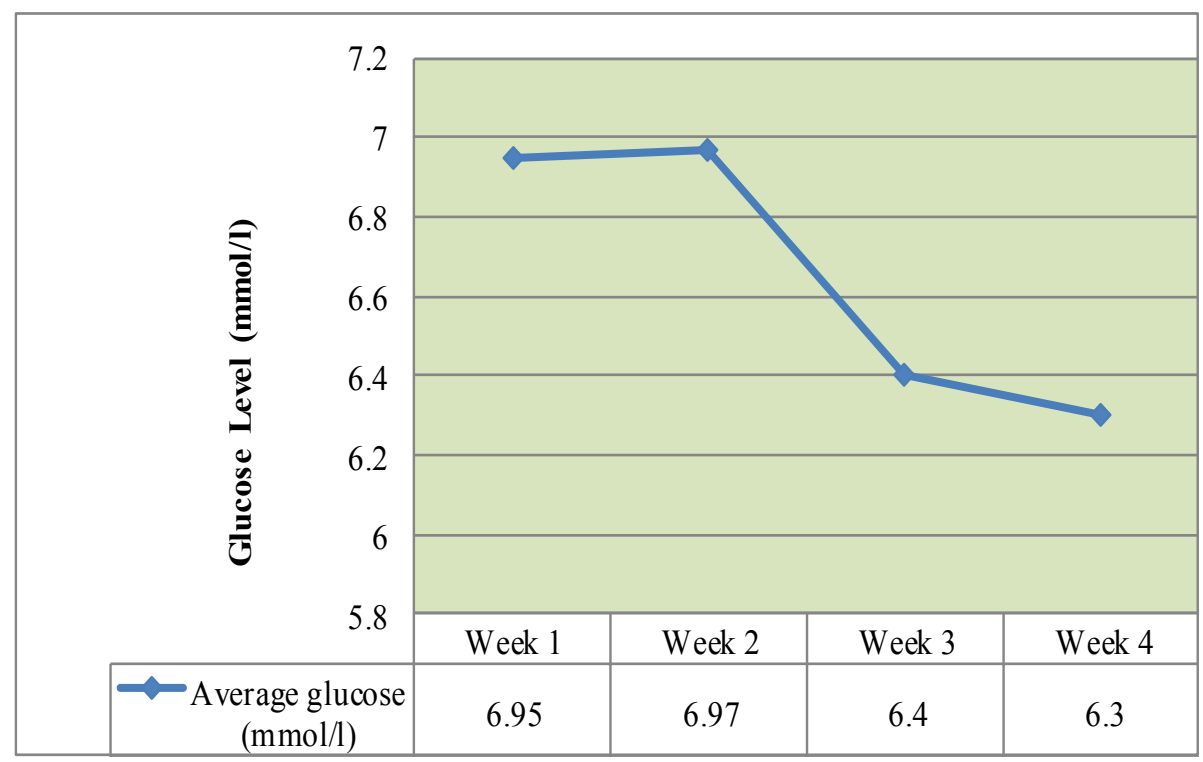

Figure 4: Average daily glucose level for each week of Ramadan

and $\mathrm{HbA}_{1 \mathrm{c}}$ was also not significant. They postulated that the improvement in glycaemic indices was due to reduced caloric intake during Ramadan and increased physical activities by performing tarawih prayer at night. Other older studies done on nondiabetic pregnant women during Ramadan fasting reported improvement in glucose control as reflected by reduction in glycaemic indices. ${ }^{13-15}$

The finding of worsened glycaemic control during Ramadan fasting was comparable with an old study done on non-pregnant diabetic populations using oral anti-diabetic agents. ${ }^{2}$ The study was done in the same locality with our study with reported significant increase up to $14 \%$ in consumption of refined sugar during the month of Ramadan. Even though the total caloric intake was reduced, the participants had increased their intake of dates and 'kueh', ie traditional Malay sweetened desserts. The blood glucose level was higher during Ramadan fasting, but overall glycaemic control was improved, shown by lower serum fructosamine level during fasting period.

We believe the reason for higher glucose level in Ramadan compared to prior to fasting is due to the technical difference in the timing of blood sugar profile monitoring. For fasting period, timing of blood sugar profile (BSP) need to be adjusted according to change in main meals time and we decided to monitor two 2-hours post-prandial level, both at sahur and iftar. The overall daily glucose level might had been higher compared to nonfasting level before Ramadan because BSP during non-fasting was taken mainly pre-prandially rather than post-prandially. This lack of standardization in the study design in term of BSP timing before Ramadan was one of our limitations, knowing that most of the patients had been monitored by health clinic prior to referral to our centre. In health clinic 
setting, the pre-prandial BSP was taken prior to lunch and dinner and prior to bed in addition to fasting level pre-breakfast.

Serum fructosamine was not used routinely in our antenatal clinic and we postulated that the test could be the better indicator for glycaemic control, especially in this kind of study, where difference in meal and BSP timing was the technical difficulty. We would like to recommend further study to incorporate serum fructosamine or even continuous glucose monitoring (CGM) technology for a more precise comparison.

Another possible explanation for higher glucose level during month of Ramadan was increase in food intake during Ramadan. Though we did not evaluate the food intake in this study, previous studies had supported this assumption. A survey done in Pakistan had reported nearly half of pregnant women claimed that they had increased food intake during Ramadan. ${ }^{6}$ A study on diet pattern changes concluded increase intake of fruit, milk and fat by $35.8 \%, 16.5 \%$ and $16.3 \%$ respectively in women during Ramadan fasting. ${ }^{16}$ In Malaysia, similar to most of other Muslim communities worldwide, increase consumption of dry dates (locally known as 'kurma') had become a tradition and this practice include among pregnant women. Dry dates contain in average amount of 70 grams of sugars per edible 100 grams, mainly in the form of simple sugars; glucose and fructose. ${ }^{17}$ Consumption of dates together with large amount of complex carbohydrates certainly has detrimental effect to glycaemic control among diabetic patients. Observation done on diabetic patients during Ramadan fasting showed the carbohydrate loads for both sahur and iftar largely exceed the total body glucose uptake capabilities (2 $\mathrm{mg} / \mathrm{kg} / \mathrm{min}$ ) for healthy individuals. ${ }^{18}$ Specifically in Malaysia, there are increased number of "iftar buffet" in hotels and "bazaar" in streets providing variety of food and drinks after long hours of fasting. ${ }^{19}$ The implications of these two phenomena which create an abundant food environment in addition to late night snack (moreh) may lead to binge eating and overeating, worsening the glycaemic control in diabetic patients.

As expected, the lowest glucose level will be towards mid-afternoon and it significantly correlated with risk of hypoglycaemia. ${ }^{20}$ In our study, the glucose level at 17:30 was consistently being the lowest level for the whole day ie 4.63 $\mathrm{mmol} / \mathrm{l}$ demonstrated in Figures 2 and 3. The levels were still above threshold for hypoglycaemia that warrant patients to break their fast as suggested in latest recommendation for Ramadan fasting in diabetic patients, i.e. $3.9 \mathrm{mmol} / \mathrm{l}$ or $70 \mathrm{mg} / \mathrm{dl}^{8}{ }^{8}$

Another peculiar observation noticed in the inpatient study was the consistent peaks in glucose levels in post-prandial time, both after sahur and iftar, as demonstrated in Figure 2 . The same observation pattern had been demonstrated in previous studies involving non-pregnant diabetic patients during Ramadan fasting. Glucose level peaks occurred in both post-prandial time in a multicentre study including Malaysia involving diabetic patients receiving glargine and glimepiride ${ }^{20}$ and prospective study in Pakistan, involving type 1 and 2 diabetic patients. ${ }^{21}$ In the study by Diabetes and Ramadan Study Group, the blood glucose levels were measured 8 times a day, even though the timing was slightly different from our current study. The graph plotted in the study resembles closely to our graph with two postprandial peaks and lowest dip during pre-iftar period. ${ }^{20}$ The possible explanation for the high peak in post-prandial glucose level especially during iftar might be due to increase in carbohydrate meal and consumptions of dates and sweet drinks during that meal. It is a common practice among majority of Muslims around the world to consume dates for iftar meal.

In our study, glycaemic control was measured using BSP in which capillary blood glucose measured four times in a day, for four days in the whole month of Ramadan; one day in each week. The BSP was done one or two days prior to clinic visit and the insulin was adjusted accordingly, aiming for optimum glucose level according to target value. ${ }^{10}$ The timing of BSP was modified for Ramadan fasting:

\section{4:00 : reflecting the pre-sahur level, after a period of short overnight fast \\ 08:00 : 2.5-hours post-prandial value, after taking sahur meal \\ 17:00 : late afternoon level, anticipating the lowest glucose level throughout the day during fasting period \\ 20:00 : 2-hours post-prandial value, after iftar meal. Usually, iftar meal will be the main and largest meal during Ramadan fasting month}

Even though the glycaemic control worsened as evidenced by higher glucose level in month of Ramadan compared to pre-Ramadan, there was an improvement in the level by the end of Ramadan compared to the beginning as shown in Figure 4. This finding was consistent with previous study done for pregnant women with diabetes who fasted during Ramadan using one type of insulin. ${ }^{12}$ The improvement in glycaemic control by the end of Ramadan was probably due to reduction in caloric intake and better diet modification after counselling given during each weekly antenatal visit. The patients might be in the state of fear and worry about risk of hypoglycaemia during beginning of fasting, so that they increased their amount of food and sweet drinks during the two main meals. Further study is needed to evaluate this assumption and to distinguish whether other factor such as physiological adaptation to prolonged fasting may play a role in the improvement in glycaemic control. 


\section{CONCLUSION \& RECOMMENDATION}

This study suggests that pregnant patients with diabetes on insulin can fast safely in Ramadan. In our group of patients, glycaemic control was relatively poorer during Ramadan fasting compared to prior to Ramadan. However, there was significant improvement in glycaemic control by the end of Ramadan, compared to the control in early of the month. There was no hypoglycaemic event in all of the patients during Ramadan fasting. The suggested insulin regimen can be safely used in properly selected and counselled pregnant women with diabetes who wish to fast during Ramadan.

\section{REFERENCES}

1. Kampan N, Azman H, Izzat Hafiz HM, Yee CS, Ghani NAA, Azlin N, et al. Outcome of pregnancy among malaysian women with diabetes mellitus-a single centre experience. Malaysia Journal of Public Health Medicine. 2013;13(2):1-10.

2. Mafauzy M, Mohammed W, Anum M, Zulkifli A, Ruhani A. A study of the fasting diabetic patients during the month of Ramadan. Med J Malaysia. 1990;45(1):14-7.

3. Salti I BE, Detournay B, for EPIDIAR Study Group. A Population-based study of diabetes and its characteristics during the fasting month of Ramadan in 13 Countries. Diabetes Care. 2004;27(10):2306-11.

4. Salleh $\mathrm{H}$. Ramadan fasting among pregnant women in Muar district, Malaysia and its association to health outcomes. Malaysian Journal of Reproductive Health: a publication of the Reproductive Research Centre of the National Population and Family Development Board, Malaysia. 1989;7 (1):69.

5. Joosoph J, Abu J, Yu S. A survey of fasting during pregnancy. Singapore Medical Journal. 2004;45(12):583-6.

6. Mubeen SM, Mansoor S, Hussain A, Qadir S. Perceptions and practices of fasting in Ramadan during pregnancy in Pakistan. Iranian Journal of Nursing and Midwifery Research. 2013;17(7).

7. Al-Arouj M, Assaad-Khalil S, Buse J, Fadhil I, Fahmy M, Hafez $S$, et al. Recommendations for management of diabetes during Ramadan: update 2010. Diabetes Care. 2010;33(8):1895-902.

8. Ibrahim M, Al Magd MA, Annabi FA, AssaadKhalil S, Ba-Essa EM, Fahdil I, et al. Recommendations for management of diabetes during Ramadan: update 2015. BMJ Open Diabetes Research \& Care. 2015;3 (1):e000108.

9. Malaysian Endocrine \& Metabolic Society. Practical Guide to Diabetes Management in Ramadan: Ministry of Health Malaysia; 2015. [online]. Available at: http:// www.mems.my. Accessed March 21, 2017.
10. Malaysia Ministry of Health. Management of Type 2 Diabetes Mellitus (5th Edition) 2015. [online]. Available at:

http://www.moh.gov.my. Accessed March 21, 2017.

11. Azlin N, Mohamed I, Adam R, Sufian SS, Wahab NA, Mustafa N, et al. Safety and tolerability of once or twice daily neutral protamine hagedorn insulin in fasting pregnant women with diabetes during Ramadan. Journal of Obstetrics and Gynaecology Research. 2011;37(2):132-7.

12. Ismail NAM, Raji HO, Wahab NA, Mustafa N, Kamaruddin NA, Jamil MA. Glycemic control among pregnant diabetic women on insulin who fasted during Ramadan. Iranian Journal of Medical Sciences. 2011;36(4):254.

13. Malhotra A, Scott P, Scott J, Gee H, Wharton B. Metabolic changes in Asian Muslim pregnant mothers observing the Ramadan fast in Britain. British Journal of Nutrition. 1989;61(03):663-72.

14. Prentice A, Prentice A, Lamb W, Lunn P, Austin S. Metabolic consequences of fasting during Ramadan in pregnant and lactating women. Human Nutrition Clinical Nutrition. 1983;37(4):283-94.

15. Dikensoy E, Balat O, Cebesoy B, Ozkur A, Cicek H, Can G. The effect of Ramadan fasting on maternal serum lipids, cortisol levels and fetal development. Archives of Gynecology and Obstetrics. 2009;279(2):11923.

16. Asar F, Hakeem R, Shaikh M, Shaikh A. Influence of ramadan fasting on dietary pattern of people with diabetes in Karachi Pakistan. Journal of the Academy of Nutrition and Dietetics. 2014;114(9):A32.

17. Alkaabi JM, Al-Dabbagh B, Ahmad S, Saadi HF, Gariballa S, Ghazali M. Glycemic indices of five varieties of dates in healthy and diabetic subjects. Nutrition Journal. 2011;10 (1):1.

18. Monnier L, Bonnet F, Colette C. New insights on glucose homoeostasis during Ramadan. Diabetes \& Metabolism. 2015;41(1):1--4.

19. Jan Mohamed HJ, Nazri NH, Loy SL. Ramadan bazaar and Ramadan buffets: The possible influence on eating behavior and health among Malaysian Muslims. Journal of Fasting and Health. 2013;1(2):43-5.

20. Salti I. Efficacy and safety of insulin glargine and glimepiride in subjects with Type 2 diabetes before, during and after the period of fasting in Ramadan. Diabetic Medicine. 2009;26(12):1255-61.

21. Ahmedani M, Haque M, Basit A, Fawwad A, Alvi S. Ramadan Prospective Diabetes Study: the role of drug dosage and timing alteration, active glucose monitoring and patient education. Diabetic Medicine. 2012;29(6):709-15. 


\section{DISCLOSURE}

The authors have no conflict of interest to be disclosed. We have no financial interests related to the material in the manuscript.

aBased on Quranic verses: "O you believed, decreed upon you is fasting as it was decreed upon those before you that you may become righteous. (Fasting for) a limited number of days. So whoever among you is ill or on a journey (during them) -then an equal number of days (are to be made up)" - AlBaqarah 2:183-184 